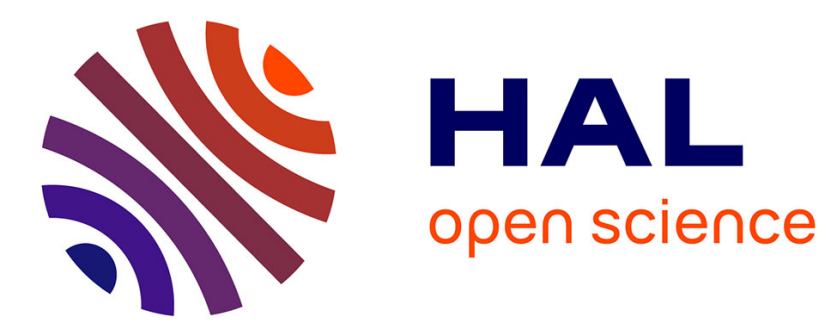

\title{
Quel rôle pour le fleuve dans le Grand Paris des marchandises?
}

\author{
Antoine Fremont
}

\section{To cite this version:}

Antoine Fremont. Quel rôle pour le fleuve dans le Grand Paris des marchandises?. Espace Géographique, 2012, 41 (3), pp. 236-251. 10.3917/eg.413.0236 . hal-02114200

\author{
HAL Id: hal-02114200 \\ https://hal.science/hal-02114200
}

Submitted on 29 Apr 2019

HAL is a multi-disciplinary open access archive for the deposit and dissemination of scientific research documents, whether they are published or not. The documents may come from teaching and research institutions in France or abroad, or from public or private research centers.
L'archive ouverte pluridisciplinaire HAL, est destinée au dépôt et à la diffusion de documents scientifiques de niveau recherche, publiés ou non, émanant des établissements d'enseignement et de recherche français ou étrangers, des laboratoires publics ou privés. 


\section{QUEL RÔLE POUR LE FLEUVE DANS LE GRAND PARIS DES MARCHANDISES ?}

Antoine Frémont

Belin | « L’Espace géographique »

2012/3 Tome 41 | pages 236 à 251

ISSN 0046-2497

ISBN 9782701175058

Article disponible en ligne à l'adresse :

https://www.cairn.info/revue-espace-geographique-2012-3-page-236.htm

Distribution électronique Cairn.info pour Belin.

(C) Belin. Tous droits réservés pour tous pays.

La reproduction ou représentation de cet article, notamment par photocopie, n'est autorisée que dans les limites des conditions générales d'utilisation du site ou, le cas échéant, des conditions générales de la licence souscrite par votre établissement. Toute autre reproduction ou représentation, en tout ou partie, sous quelque forme et de quelque manière que ce soit, est interdite sauf accord préalable et écrit de l'éditeur, en dehors des cas prévus par la législation en vigueur en France. Il est précisé que son stockage dans une base de données est également interdit. 


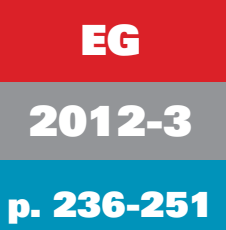

\title{
Quel rôle pour le fleuve dans le Grand Paris des marchandises?
}

\author{
Antoine FrÉmONT \\ Université Paris Est \\ Institut français des Sciences et Technologies des Transports, \\ de l'Aménagement et des Réseaux (IFSTTAR) \\ antoine.fremont@rff.fr
}

RÉSUMÉ .- Est-il possible d'envisager un rôle pour le fleuve dans le Grand Paris des marchandises, depuis les grands flux internationaux en provenance des ports maritimes jusqu'à ceux de la distribution urbaine? Le transport des marchandises participe aux logiques métropolitaines. La force du système routier aboutit à une double déconnexion entre le fleuve et la ville, déconnexion par rapport à la production et à la distribution accompagnée d'une déconnexion spatiale, mise en évidence par le processus de " périurbanisation logistique ». Paris, métropole de premier rang et carrefour européen, fournit un terrain exemplaire. Les pistes de reconnexion ne peuvent se concevoir que dans une stratégie volontariste d'aménagement des territoires de la logistique métropolitaine.

AMÉNAGEMENT, FLEUVE, LOGISTIQUE, MÉTROPOLISATION, PÉRIURBANISATION, TRANSPORT DE MARCHANDISES
ABSTRACT . - What role for the Seine in the Greater Paris market?- Can the river Seine play a key role in the freight transport system in "Grand Paris" (Greater Paris), from international flows to urban distribution outlets? Firstly, a systemic approach to the freight transport system within metropolitan areas is outlined. The prominence of the road system has led to a twofold disconnection between the river and the city, a disconnection from production and distribution processes, and also a spatial disconnection highlighted by the process of "logistics suburbanization". As a major European metropolitan area, Paris provides a very useful example. The paper recommends reconnecting the river to the city for freight transport. This can only be achieved through strongwilled metropolitan planning and policies aimed at freight and logistics activities.

FREIGHT TRANSPORT, LOGISTICS, METROPOLITAN AREA, RIVER, SUBURBANIZATION, URBAN PLANNING

\section{Introduction}

De très nombreuses villes sont situées sur un fleuve. Un vaste mouvement de redécouverte de leur matrice originelle par les villes centres est actuellement à l'œuvre. Depuis la préhistoire, les activités de pêche et d'agriculture, les activités artisanales, ensuite industrielles, ou les transports ont utilisé intensément le fleuve comme une ressource essentielle. Puis, sous l'effet de l'utilisation d'autres énergies, des voitures et des camions, et de l'expansion des villes bien au-delà de leur site originel, le lien s'est distendu avec des fleuves de moins en moins naturels, réceptacles des pollutions urbaines, franchis par des ponts, cernés par des autoroutes ou des voies de chemin de fer, souvent canalisés mais ne jouant qu'un rôle marginal par rapport à la route. 
Les cours d'eau ont permis jusqu'à la Révolution industrielle d'acheminer les marchandises dans le centre des villes. Le transport fluvial peut-il aujourd'hui jouer un rôle dans l'approvisionnement des villes alors que sa part est devenue marginale et que le retour en grâce de l'eau dans les représentations est beaucoup plus synonyme de loisirs et de spéculations immobilières que d'activités marchandes, industrielles et de transport? Si oui, quelles sont les conditions à réunir? Mais pourquoi envisager un rôle pour le fleuve alors que la route remplit si bien le sien? Le développement durable, via les pollutions et autres nuisances produites par le transport routier, vient immédiatement à l'esprit comme un attendu ne souffrant plus aujourd'hui la discussion. Au-delà, ce sont les formes mêmes de la ville qui sont en question.

Envisager un rôle pour le fleuve nécessite d'abord de comprendre la force du système routier de marchandises actuel et son empreinte sur les villes. Le raisonnement mêle des échelles différentes, de la plus petite, celle des grands corridors internationaux, jusqu'à la plus grande qui s'achève avec la livraison des marchandises dans les rayons des magasins (Hesse, 2008). Au-delà de la ville elle-même, les processus de métropolisation - qui mêlent à petite échelle les forces d'agglomération et à plus grande échelle des forces centrifuges - fournissent des variables clés d'explication.

Le Grand Paris, connu avant tout pour son projet de métro en rocade, est un terrain exemplaire. C'est une métropole de premier rang et un carrefour en Europe. Elle est reliée aux grandes routes maritimes mondiales par les ports du Havre et de Rouen au débouché de l'axe Seine, considéré de façon récurrente par les politiques (Napoléon) ou les architectes urbanistes (Grumbach, 2009) comme l'axe métropolitain par excellence. Mais celui-ci est structuré depuis 60 ans par des générations successives d'autoroutes et de rocades.

Cet article explore, à travers l'exemple de la métropole parisienne, la déconnexion du transport des marchandises entre le fleuve et la métropole pour proposer ensuite des pistes de reconnexion (Hall, 2010).

\section{Fleuve, marchandises et métropolisation : la double déconnexion}

\section{Le transport fluvial: un mode déconnecté de la production et de la distribution}

La voie d'eau joue aujourd'hui pour le transport des marchandises un rôle marginal, tout comme le rail. Quelques causes simples liées à l'évolution des systèmes de production et de distribution expliquent ce grand chambardement. Les deux chocs pétroliers de 1973 et 1979 marquent la fin des Trente Glorieuses et l'achèvement d'un cycle où les industries lourdes formaient les moteurs de la croissance. La part relative des produits en vrac et des produits lourds dans le total des marchandises à transporter diminue. Or le rail ou la voie d'eau sont particulièrement bien adaptés au transport de ces produits lourds (Savy, 2006b). Pour la voie d'eau s'ajoute la fermeture progressive des complexes industriels intérieurs issus de la première révolution industrielle et situés le long des voies d'eau.

L'entrée dans une ère dite "postfordiste " modifie les méthodes de production et de distribution. Cette dernière est tirée par la demande en suivant le principe du juste à temps. Les produits, moins lourds et à forte valeur ajoutée, sont formés de multiples composants fabriqués dans des unités spécialisées afin de bénéficier des économies d'échelle. Ces unités sont dispersées géographiquement, y compris à une 
Tabl. 1/ Forces d'agglomération et de dispersion

\begin{tabular}{|l|c|c|c|}
\hline \multirow{2}{*}{ Forces } & \multicolumn{3}{|c|}{ Échelle } \\
\cline { 2 - 4 } & $\begin{array}{c}\text { Petite } \\
\text { (espace } \\
\text { national et } \\
\text { européen) }\end{array}$ & $\begin{array}{c}\text { Moyenne } \\
\text { (aire urbaine) }\end{array}$ & $\begin{array}{c}\text { Grande } \\
\text { (nœud routier) }\end{array}$ \\
\hline Agglomération & + & - & + \\
\hline Dispersion & - & + & - \\
\hline
\end{tabular}

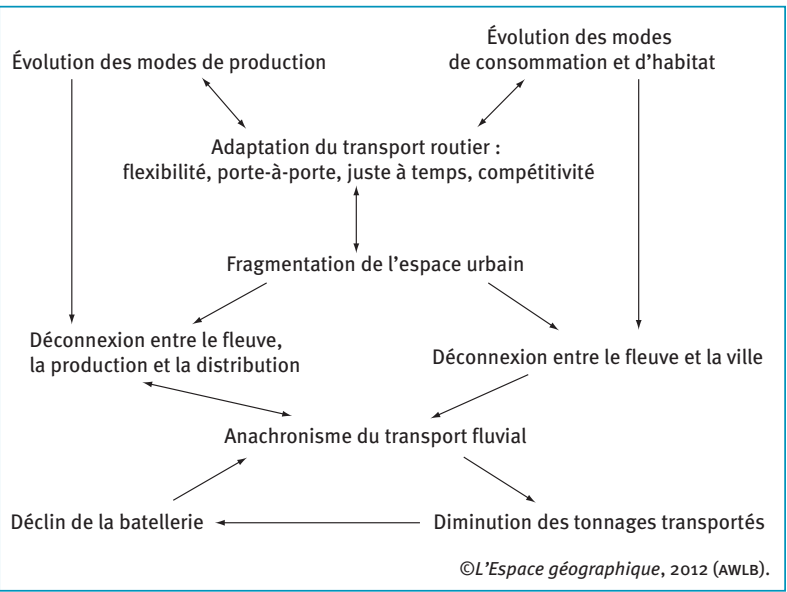

Fig. 1/ Déconnexion du fleuve et de la ville échelle internationale. La demande de transport est caractérisée par la multiplication des envois de petite taille qui s'inscrivent dans une logique de flux tendus et par leur forte dispersion à la fois dans le temps et dans l'espace (tabl. 1).

Si la route a aujourd'hui atteint en France et en Europe des parts de marché supérieures à $80 \%$, c'est qu'elle participe de ce vaste assemblage de la production et de la distribution (Dablanc, 2007). Le transport routier est adapté à la demande de transport grâce à sa très grande flexibilité, à des prix faibles expliqués par une très forte concurrence tant à l'échelle française qu'européenne et à la rapidité et à la finesse de la desserte géographique, rendue possible par le développement d'un vaste réseau autoroutier. Associés aux camions, les entrepôts sont les lieux du groupage, de l'éclatement, du tri et du stockage des marchandises. Ce système routier est devenu de fait une composante essentielle de la production et de la distribution.

Depuis les années 1970, le report modal s'opère à grande échelle, à l'inverse de tous les effets d'annonce, du rail et du fleuve vers la route. Ces deux " vieux " modes issus de la révolution industrielle sont aujourd'hui largement déconnectés des systèmes de production et de distribution. Ils ne jouent plus qu'un rôle marginal qui auto-alimente leur déclin comme en témoigne la chute drastique des effectifs de bateliers et leur vieillissement (fig. 1).

\section{Fleuve et métropole: la déconnexion spatiale}

L'évolution spatiale des villes modèle aussi le transport des marchandises en lien avec la production et la distribution. Deux forces peuvent être retenues pour expliquer la dynamique urbaine à l'échelle métropolitaine. Les forces centripètes renvoient au processus d'agglomération que représente la ville en général. Elles s'exercent d'autant plus fortement avec la métropolisation qui «se caractérise par un accroissement du poids des plus grandes villes dans la répartition de certaines fonctions, ainsi que de la concentration de la population dans des aires métropolitaines " (Elissalde, Hypergéo ${ }^{1}$ ). La ville attire. Inversement, à plus grande échelle, celle de l'aire urbaine, des forces centrifuges rejettent vers la périphérie les fonctions les plus banales pour conserver dans le cœur celles à forte valeur ajoutée (Combes et al., 2006). Elles participent à la dispersion et à l'étalement des activités en périphérie, redessinant avec des tracés de plus en plus incertains les limites floues de la métropole. Forces centripètes à petite échelle et centrifuges à grande échelle permettent de mettre en évidence trois moments logistiques liés à la dynamique urbaine (fig. 2).

Le premier moment logistique se situe chronologiquement avant la révolution industrielle. Les vallées sont des lieux privilégiés de l'implantation des villes européennes, 1.http://www.hypergeo. eu/spip.php?article75 


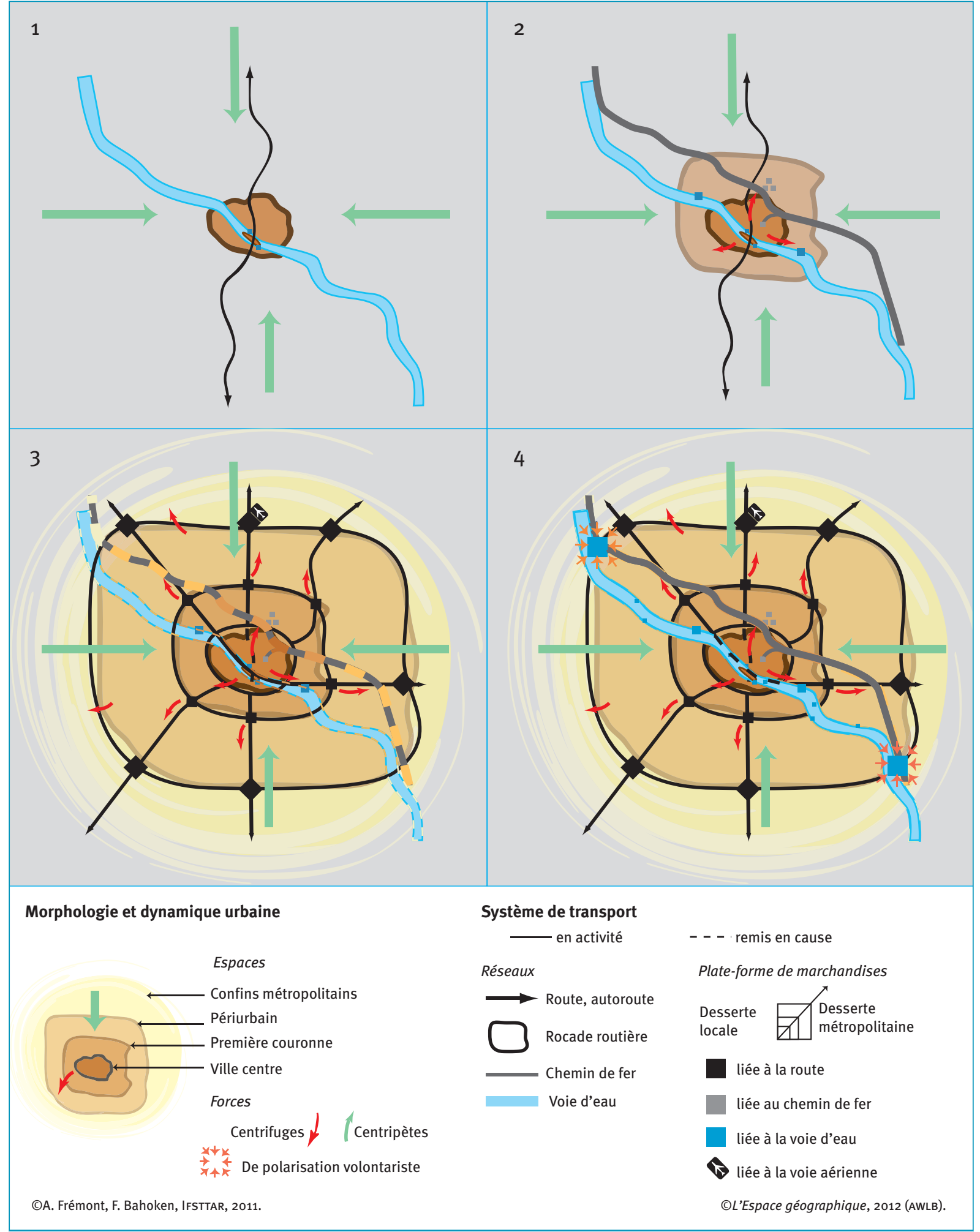

Fig. 2/ Quatre moments logistiques 
2. Depuis 2007, avec l'ouverture du fret ferroviaire à la concurrence, les données statistiques sur le fret ferroviaire manquent car les données propres à la SNCF ne peuvent être diffusées qu'agrégées aux résultats des nouveaux opérateurs. Or les statistiques relatives à l'ensemble des opérateurs ferroviaires ne sont pas disponibles. Les données complètes les plus récentes concernent donc l'année 2006. L'ouverture à la concurrence se traduit donc par une plus grande opacité du marché! avec des sites associant une route terrestre à un passage sur le fleuve. Les cours d'eau permettent le transport des marchandises vers les villes qui sont aussi des places de marché. L'activité " marchandise " s'effectue au cœur de la ville, directement sur des berges d'échouage, ou dans les plus grandes villes, comme à Paris aux XVII et $\mathrm{XVIII}^{\mathrm{e}}$ siècles, le long de véritables quais qui sont alors, selon l'expression de Pierre de l'Estoile, la "clé des vivres de Paris " (cité par Lalandre, 2004).

Le deuxième moment logistique s'étend de la Révolution industrielle à l'avènement du transport routier, du XIX ${ }^{\mathrm{e}}$ siècle aux Trente Glorieuses. Avec l'essor urbain, les forces centrifuges s'exercent. Le centre n'est pas capable d'accueillir toutes les activités en son sein. Une première couronne se développe autour des villes. Elle mélange habitat et activités industrielles. La voie d'eau, renforcée par le développement des canaux, reste un lieu privilégié de l'implantation industrielle, comme le montre l'exemple de Strasbourg (Beyer, Debrie, 2011). Le port fluvial prend des dimensions industrielles, incompatibles avec une localisation centrale. Cependant, une première déconnexion entre le fleuve et la ville se produit avec l'arrivée du chemin de fer. Concurrent direct de la voie d'eau, il offre une alternative pour l'implantation des activités industrielles ou l'approvisionnement urbain.

La déconnexion radicale entre la ville et le fleuve correspond au troisième moment logistique, entièrement dominé par le système routier qui se met en place avec les Trente Glorieuses. À petite échelle, les forces centripètes continuent de l'emporter: les plus grandes villes concentrent les entrepôts parce qu'elles sont les plus grands centres de production et de consommation tout en étant très bien connectées aux autres villes grâce au développement du réseau autoroutier (Cidell, 2010). À l'échelle de l'aire urbaine, le système routier crée un effet de levier en faveur des forces centrifuges. Les zones périphériques, où les prix du foncier diminuent avec l'éloignement par rapport au centre, gagnent en accessibilité grâce au développement du réseau autoroutier. Les fonctions logistiques sont rejetées à l'extérieur (Dablanc, Rakotonarivo, 2010). Dans la première couronne, elles sont aussi en concurrence avec le développement des centres commerciaux et tertiaires. Elles sont rejetées dans la couronne périurbaine où des espaces sont encore disponibles pour de vastes entrepôts qui répondent à la demande logistique actuelle. À grande échelle, ces entrepôts sont situés à proximité des échangeurs autoroutiers et le long des rocades qui ceinturent les villes afin de bénéficier d'une forte accessibilité en coût et en temps. Un phénomène similaire est observé aux États-Unis (Bowen, 2008). Les forces centrifuges aboutissent à un mitage logistique et à une dilatation de l'espace métropolitain avec un processus d'agglomération le long des axes routiers qui jouent alors à l'échelle de l'aire urbaine un rôle structurant. La logistique, tout comme l'habitat, participe à l'étalement de la métropole qui s'accompagne de la création de nouveaux pôles d'activités en périphéries, donnant à la métropole son caractère polycentrique. Les processus étant très similaires à celui de l'habitat, le terme de "périurbanisation logistique » est proposé pour désigner ce troisième moment logistique.

\section{Le Grand Paris des marchandises dominé par le " tout routier "}

En $2006^{2}$, les flux de trafic créés par l'île-de-France s'élèvent à 296 millions de tonnes, soit environ $12 \%$ des trafics de la France entière. Alors $47 \%$ de ces flux se réalisent à l'intérieur de la région, $39 \%$ avec les autres régions françaises et $14 \%$ à 
l'international; $84 \%$ de ces trafics s'effectuent par la route et cette part monte à $90 \%$ pour les volumes échangés entre l'île-de-France et le reste de la France métropolitaine. À cela, il faut ajouter des trafics de transit routier estimés à 64 millions de tonnes (DREIF, 2007). L'organisation logistique de la métropole parisienne est déconnectée du fleuve, entièrement structurée par la route. La voie d'eau ne joue qu'un rôle de niche.

\section{L'effet structurant de la logique routière sur la métropole}

L'évolution de la localisation des entrepôts en Île-de-France permet de mettre en évidence la "périurbanisation logistique ». Dans les entrepôts sont essentiellement traités des produits manufacturés et de la messagerie ainsi que des produits alimentaires qui représentent respectivement $31 \%$ et $9 \%$ des tonnages échangés en Île-de-France en 2006. Destinés à la consommation finale, ces produits nécessitent presque systématiquement le passage par un entrepôt pour des opérations de groupage-dégroupage, stockage ou reconditionnement (Guilbaut, 2008). La part de la route est supérieure à $96 \%$ pour leur transport. Ces deux catégories de produits renvoient directement au fonctionnement métropolitain actuel.

La localisation des entrepôts a été appréhendée à la commune via les permis de construire connus par la base de données SitADEL du MEEDEM (fig. 3) ${ }^{2}$. Un cumul sur des périodes de cinq ans depuis 1980 permet de mettre clairement en évidence la dynamique spatiale. Le premier constat est celui de l'étalement logistique. On construit des entrepôts de plus en plus loin de Paris. Dans les années 1980, la première couronne reste un lieu privilégie d'implantation. Vient ensuite un processus de desserrement qui implique les départements de la deuxième couronne (tabl. 2). Il se traduit par une modification dans les "axes logistiques ». Dans les années 1980, deux axes ouest-sudouest/est-nord-est se situent de part et d'autre de Paris, l'un dans le Nord, l'autre dans le Sud, essentiellement le long de l'A86 alors en construction. L'axe au nord correspond à l'ancienne banlieue industrielle de Paris avec un étirement progressif des localisations d'entrepôts vers l'aéroport de Roissy. Au nord-ouest, le pôle de Cergy, notamment avec le parc logistique situé sur la commune de Saint-Ouen-l'Aumône, ne cesse de prendre de l'ampleur, profitant de la Francilienne qui lui donne une forte accessibilité à Roissy. Une évolution majeure se produit dans les années 1990. L'axe se décale nettement vers l'est pour devenir nord-sud, le long de la Francilienne, de Roissy jusqu'à Évry/CorbeilEssonnes.

$\mathrm{Au}$ total, l'observation de la carte du parc logistique francilien en 2009 (fig. 4) permet de mettre en évidence un axe nord-est/sud-ouest qui va de l'A1 au nord à l'A6 et à l'A10 au sud et qui court le long de l'A86 et de la Francilienne, cette dernière devenant le lieu privilégié de la localisation des nouveaux entrepôts.

Un triple jeu d'échelles explique ce changement. Aux échelles européenne et nationale, la Francilienne appartient à l'axe européen qui met en relation l'Europe du Nord avec l'Europe du Sud. L'Île-de-France est aussi à l'échelle nationale un carrefour essentiel. À cette échelle jouent à plein les effets d'agglomération. De fait, l'Île-de-France concentre à elle seule $25 \%$ des surfaces d'entrepôts en France. Le couloir nord-sud de la Francilienne permet aux chargeurs et/ou transporteurs de répondre aux besoins du très vaste marché local et d'assurer un rôle de redistribution des marchandises aux échelles nationales et européennes.

À l'échelle de la métropole, cette localisation le long de la Francilienne et des radiales autoroutières traduit le mouvement de desserrement et de périurbanisation
2. La principale faiblesse de cette approche est l'impossibilité d'identifier les destructions d'entrepôts ou leur changement d'affectation, notamment lorsque des surfaces construites sont cumulées dans la longue durée. Les travaux effectués par la DrEIF (Graille, 2009) pour affiner l'estimation du parc des entrepôts franciliens, données malheureusement non publiques, ne remettent pas en cause les tendances lourdes indiquées par cet article. 


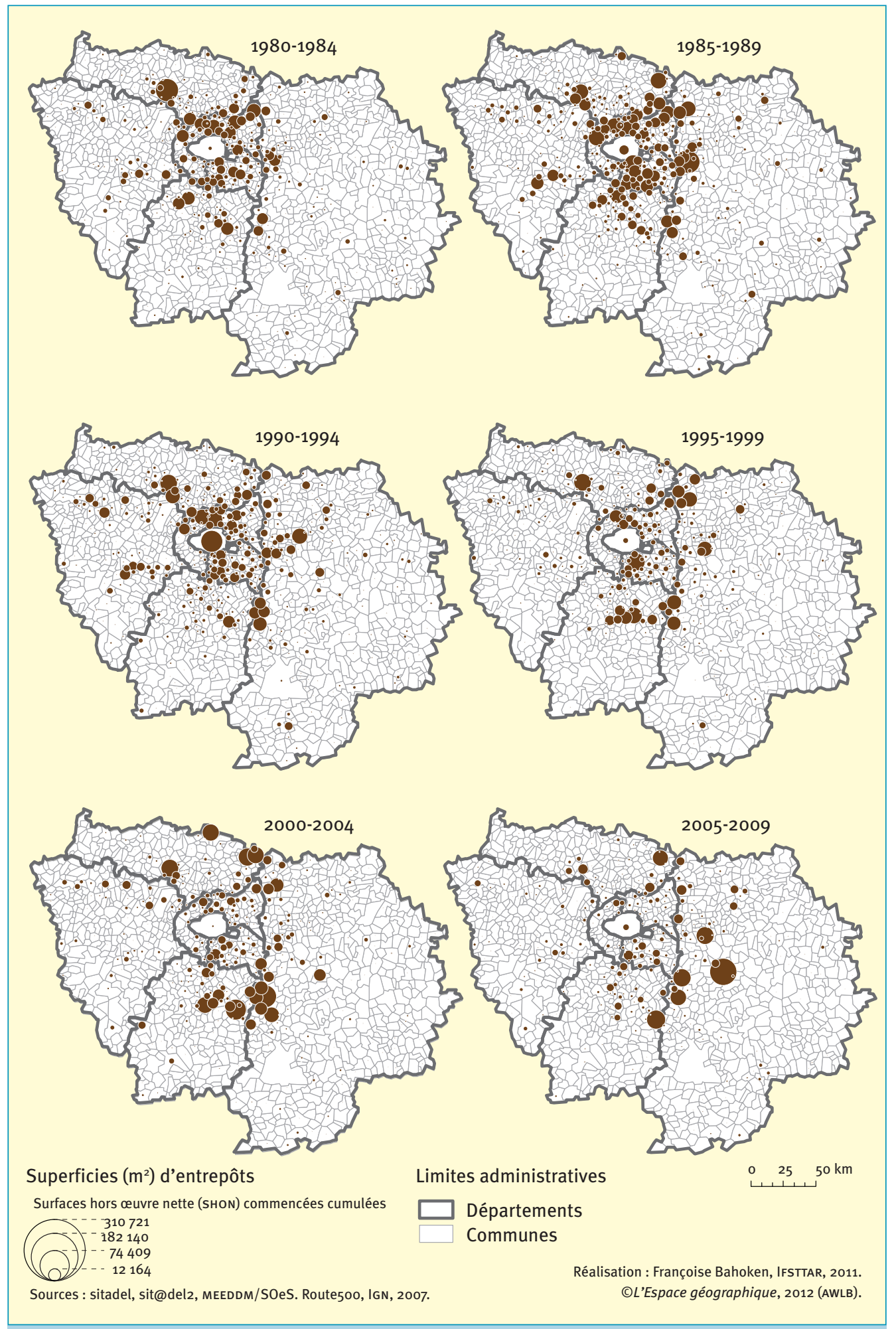

Fig. 3/ Évolution de la localisation des entrepôts neufs en île-de-France entre 1980 et 2009 
logistique. Elle pérennise une opposition ouest-est qui est aussi sociale. L'ouest de la métropole parisienne est un véritable désert logistique, à l'exception de quelques grappes isolées le long de l'autoroute de Normandie, ce qui est très défavorable aux ports du Havre dont le marché principal s'éloigne en distance temps.

À très grande échelle, les effets d'agglomération sont à nouveau à l'œuvre. Les entrepôts sont localisés d'une façon privilégiée à l'intersection de l'axe nord-sud (la Francilienne) et des autoroutes qui partent en radiale de Paris vers la Province. C'est la recherche d'une accessibilité maximum, à toutes les échelles, aussi bien pour desservir le supermarché local qu'une usine située à l'autre bout de la France, qui explique ces localisations. De fait, $75 \%$ des surfaces d'entrepôts se concentrent dans cinq grandes zones avec un grand rôle joué par les villes nouvelles (tabl. 3). La logistique participe à l'émergence de nouvelles centralités en périphérie et à donner à la métropole francilienne son caractère polycentrique. Au-delà

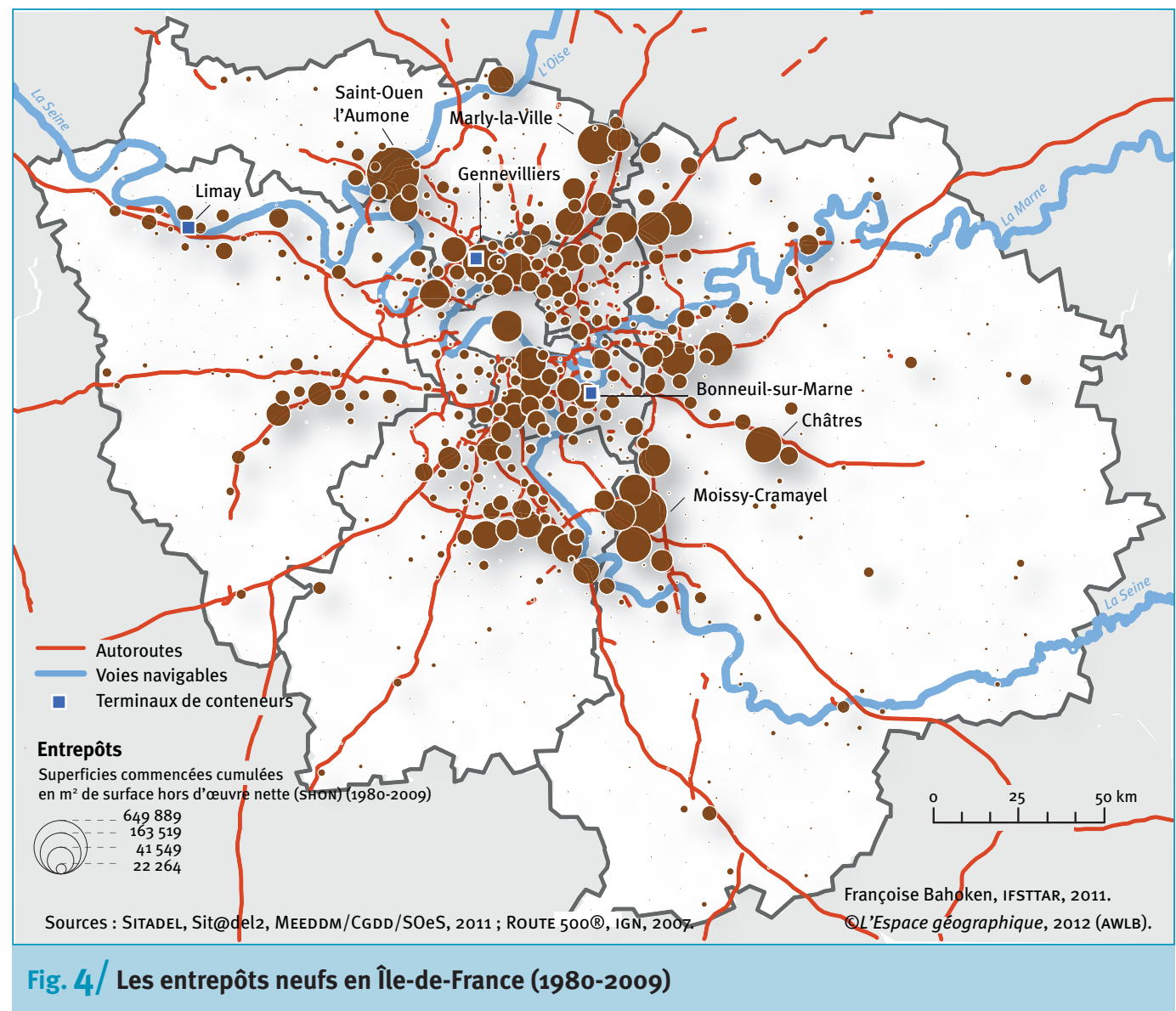


Tabl. 3/ Les grandes concentrations

d'entrepôts en île-de-France dont les villes nouvelles

\begin{tabular}{|l|c|}
\hline Territoire & Pourcentage \\
\hline Roissy & 17 \\
\hline Sénart-centre Essonne & 17 \\
\hline \multicolumn{1}{|c|}{ dont Sénart } & 8 \\
\hline Plaine de France-Gennevilliers & 15 \\
\hline Seine Amont-Nord Essonne & 15 \\
\hline \multicolumn{1}{|c|}{ dont Cergy-Pontoise } & 4 \\
\hline Cergy & 5 \\
\hline & 4 \\
\hline Marne-la-Vallée & 5 \\
\hline Saint-Quentin-en-Yvelines & 75 \\
\hline Total & \\
\hline Source: Graille, 2009. & \\
\hline
\end{tabular}

de ces quatre zones essentielles, chaque sortie d'autoroute est un lieu potentiel de localisation d'entrepôts, l'attractivité de la sortie d'autoroute diminuant au fur et à mesure de son éloignement de Paris.

La voie d'eau et les ports:

un rôle de niche dans une logique métropolitaine

La prépondérance de la route est telle pour le transport des marchandises que la voie d'eau ne peut jouer en Île-de-France qu'un rôle marginal, tout comme le rail. En 2006, 16 millions de tonnes ont été transportées par voie d'eau, soit $6 \%$ des trafics de l'Île-de-France. Cette part est supérieure à celle du rail (12 Mt, 4 \%). Elle n'a pas cessé de diminuer depuis les années 1970 pour atteindre un minimum dans le courant des années 1990 et progresser à nouveau depuis lors, mais sans retrouver les niveaux record des années 1970. Les Ports de Paris traitent un trafic d'environ $20 \mathrm{Mt}$ depuis les années 2000 alors que celui-ci atteignait 42 Mt en 1969.

La voie d'eau a aujourd'hui une place particulière sur le marché du transport. Elle est spécialisée dans l'acheminement des produits en vrac. Les matériaux de construction, les produits agricoles, les minerais et les déchets, les produits pétroliers et les combustibles solides représentent plus de $90 \%$ de l'activité de la voie d'eau. La voie d'eau permet d'exporter les productions céréalières vers le port de Rouen. À l'inverse, elle joue un rôle non négligeable dans l'acheminement des produits énergétiques en provenance du port du Havre mais surtout un rôle prépondérant dans l'acheminement des matériaux de construction en provenance de la Haute-Normandie, de la Picardie et de la région Île-de-France elle-même. La complémentarité avec la route est évidente. Plus de la moitié des trafics internes à la région sont des matériaux de construction transportés à plus de $93 \%$ par la route mais sur des distances très courtes, inférieures à $27 \mathrm{~km}$, alors que ces mêmes matériaux de construction représentent plus de $90 \%$ des trafics de la voie d'eau à l'intérieur de la région Île-de-France, mais sur des distances moyennes de plus de $80 \mathrm{~km}$. La voie d'eau est utilisée dans la partie la plus longue du trajet, les camions permettant d'effectuer les derniers kilomètres pour atteindre les chantiers. Les très nombreux sites portuaires multiplient les possibilités de chargement/déchargement, y compris dans ou à proximité immédiate de Paris intra-muros, avec une réelle complémentarité entre les différents sites (Paffoni, 2011). Par exemple, à l'ouest de Paris, le port d'Issy-les-Moulineaux traite près d'un million de tonnes par an.

Dans ce marché de niche, l'axe Seine est essentiel: $40 \%$ des trafics des Ports de Paris ont pour origine ou destination la Haute-Normandie et $46 \%$ la région Île-deFrance elle-même. La Picardie et la Bourgogne arrivent loin derrière avec un peu plus de $3 \%$ des trafics chacune. Il est vrai que vers la Bourgogne comme vers la Picardie, les gabarits se réduisent brutalement, passant de 3000 tonnes à 650 tonnes pour le canal du Nord et l'Yonne et au gabarit Freycinet (moins de 400 tonnes) pour les canaux allant vers le sud. La Seine est un bassin enclavé.

À cette logique métropolitaine de niche de la voie d'eau s'ajoute le rôle essentiel des espaces portuaires comme lieux d'implantation logistique. Les Ports de 
Paris gèrent 1 million de $\mathrm{m}^{2}$ d'entrepôts répartis sur une dizaine de sites principaux, soit près de $6 \%$ du parc francilien. Le port de Gennevilliers en concentre à lui seul $30 \%$. Bonneuil-sur-Marne et Limay sont les deux autres sites les plus importants. Gennevilliers comme Bonneuil forment un héritage. Ils ont été conçus dans les années 1920 comme des ports fluviaux modernes avec des darses et des quais à un moment où la voie d'eau jouait un rôle essentiel. Bonneuil est très actif pendant la Première Guerre mondiale pour alimenter le front à l'est. Gennevilliers connaît son apogée dans les années 1950. Cet héritage se révèle une aubaine dans la logique métropolitaine actuelle. Il permet à des entreprises de messagerie, de la distribution et de l'automobile de disposer d'entrepôts et/ou de surfaces de stockage dans le cœur de l'agglomération et aux Ports de Paris de vivre aisément, non pas du trafic fluvial, mais de la rente foncière grâce aux revenus de la location des entrepôts. Très logiquement, les trafics de ces ports sont essentiellement routiers.

\section{Intégrer le fleuve dans les logiques métropolitaines de transport}

\section{Une fenêtre d'opportunité}

Une fenêtre d'opportunité existe aujourd'hui en faveur des "vieux " modes qui prennent alors, par un retournement sémantique, la dénomination de "modes alternatifs ". L'enjeu environnemental est le plus à la mode, celui apparu aussi le plus récemment. Les arguments sont biens connus. Le transport routier de marchandises pollue fortement. Si l'on s'en tient aux seules émissions de gaz à effet de serre, le transport est le premier secteur émetteur en France avec un quart des émissions, les poids lourds participant à hauteur de $27 \%$ de ces émissions et leur part ne cessant d'augmenter. En Île-de-France, 80000 poids-lourds entrent et sortent chaque jour des 17 péages de la région. Avec des intérêts différents, les pouvoirs publics, l'opinion via les diverses associations et, dans une moindre mesure, les entreprises partagent cet enjeu devenu commun.

La congestion routière entrave la circulation. Elle affecte principalement les aires urbaines. Pour les entreprises, elle remet en cause la fiabilité des chaînes de transport, indispensable à celle des systèmes de production et de distribution. Pour les automobilistes, les camions sont perçus, sans doute à tort, comme les principaux responsables de cette congestion. La congestion routière est une entrave au développement des activités économiques. Ce crédo de la compétitivité par les transports a longtemps été porté comme enjeu unique, parce que jugé déterminant, par les milieux économiques et les pouvoirs publics (État, Union européenne), pour justifier les investissements en infrastructures, notamment dans le domaine routier, mais avec une tendance récente en faveur du rail et/ou de la voie d'eau. 
Troisième facteur: le rail ou la voie d'eau permettent de transporter de grandes quantités de marchandises à bas prix. L'effet de ces modes dits "massifiés " se fait d'autant plus sentir que les distances parcourues sont longues. Les ports maritimes sont des points essentiels d'aboutissement ou de départ de ces corridors car ils accueillent de grands volumes de marchandises, de plus en plus en relation avec la conteneurisation. Ces corridors sont aussi un moyen pour ces ports d'élargir leur hinterland d'autant qu'ils s'inscrivent dans la politique des réseaux transeuropéens (RTE) qui, malgré ses déficiences, influence fortement les politiques nationales d'infrastructures. Ne pas faire partie de ces réseaux transeuropéens est synonyme d'un risque de marginalisation des territoires et de perte de compétitivité. Les corridors de transport massifiés qui relient les ports maritimes aux grandes métropoles intérieures deviennent un facteur de la compétition entre les ports et de l'insertion dans la mondialisation (O'Connor, 2010).

Ces trois facteurs ont rendu possible la mise en place de voies ferroviaires et fluviales. Transporteurs, chargeurs, pouvoirs publics, associations environnementales ou de riverains se retrouvent avec des intérêts totalement différents autour d'un objectif commun, celui du développement des modes dits alternatifs. Le Grenelle de l'environnement a illustré cette fenêtre d'opportunité. La décision de construire le canal Seine-Nord-Europe en est une des traductions concrètes même si les financements nécessaires ne sont pas encore aujourd'hui réunis.

\section{Quelles leçons tirer de l'essor du trafic conteneurisé fluvial sur la Seine?}

Sans entrer dans des scénarios fictions avec le canal Seine-Nord Europe, il est intéressant de tirer les leçons de l'essor du trafic fluvial conteneurisé sur la Seine entre le port du Havre et le terminal de Gennevilliers, qui concentre à lui seul $85 \%$ des trafics conteneurs des Ports de Paris. Rien a priori ne militait en faveur de la voie d'eau: rapidité du camion d'un côté, lenteur de la barge de l'autre, accentuée par les méandres de la Seine; transport direct du terminal maritime à la destination finale avec le camion, versus des ruptures de charge et la contrainte d'un trajet routier pour les derniers kilomètres, du terminal intérieur à la destination finale. Pourtant, depuis le début des années 2000, le trafic fluvial ne cesse de se développer, y compris pendant les deux dernières années pourtant marquées par la crise. La part de la voie d'eau dans les trafics conteneurisés d'hinterland du port du Havre s'établit aujourd'hui à plus de $9 \%$ alors qu'elle était encore quasi nulle au milieu des années 1990.

Ce succès s'explique par la réunion de conditions nécessaires au développement de ce type de transport (Frémont et al., 2009; Frémont, Franc, 2010). Le service proposé au chargeur doit être un service porte à porte, du terminal portuaire à l'entrepôt. Cela suppose un opérateur de transport qui coordonne tous les maillons et intervenants de la chaîne de transport: manutention portuaire, transport fluvial, manutention sur le terminal intérieur, pré- et post-acheminements routiers. Un intégrateur de transport est responsable de la mise en place de ce service de transport combiné. C'est le métier par exemple des sociétés Logiseine ou River Shuttle Container (RSC).

Le prix porte à porte doit être inférieur de 10 à $20 \%$ par rapport à celui de la route afin de créer un effet de levier en faveur du transport combiné. La massification du transport par la voie d'eau permet d'obtenir ce prix inférieur. Des convois de deux barges contenant chacune $192 \mathrm{EVP}$ sont possibles sur la Seine. Tout ce qui tend à renchérir le coût du transport terrestre, notamment routier, comme une augmentation des 
prix du gasoil, une pénurie de l'offre ou un renforcement de la législation sociale, donne un avantage comparatif aux transports massifiés et, inversement, une baisse des prix du transport routier, par exemple liée à la crise économique, leur est très défavorable.

Les services fluviaux doivent proposer une fréquence suffisante et une fiabilité identique, voire supérieure à celle de la route. Ce n'est pas tant la durée du transport qui compte que la possibilité d'insérer les flux de marchandises dans les rythmes juste à temps de la production et/ou de la distribution. Le temps de parcours et le stationnement des conteneurs sur le terminal fluvial donnent une latitude supplémentaire pour ajuster le délai de livraison.

Des avantages innovants peuvent aussi être trouvés, comme la possibilité de dédouaner la marchandise au port fluvial, voire dans les entrepôts, et non au port maritime. Cela permet au chargeur de réaliser des économies confortables de trésorerie. Enfin, la voie d'eau permet aux chargeurs et/ou aux transporteurs d'associer, dans l'esprit des consommateurs, leurs enseignes avec la notion de développement durable.

Les grandes compagnies maritimes ont joué un rôle décisif dans le développement des trafics fluviaux conteneurisés parce qu'elles y avaient un intérêt: assurer des flux de conteneurs entre Le Havre et la région parisienne, l'hinterland le plus riche et le plus proche du port maritime, afin de remplir leurs très gros navires, tirer les prix terrestres vers le bas en jouant sur la massification des flux dans l'hinterland afin d'accroître leurs parts de marché, ce qui engendre une concurrence vertueuse, chacun imitant l'autre.

À cet intérêt des compagnies maritimes a répondu celui des très gros chargeurs de la grande distribution. Des enquêtes menées par l'Institut d'Aménagement et d'Urbanisme de la région d'Île-de-France en 2007, 2008 et 2009 sur les terminaux de Gennevilliers et Bonneuil-sur-Marne montrent que plus de $80 \%$ des trafics sont liés à une dizaine de chargeurs qui alimentent leurs supermarchés en produits venus d'Asie orientale (IAURIF, 2008). Elles révèlent aussi que la destination des conteneurs à partir de Gennevilliers se calque sur celle des entrepôts de ces grands chargeurs, principalement localisés dans le département de la Seine-et-Marne qui capte à lui seul plus de la moitié des conteneurs pleins (fig. 5). De très longs pré- et post-acheminements routiers de $50 \mathrm{~km}$ et plus, en zone urbaine dense, sont nécessaires pour atteindre à partir du terminal de Gennevilliers les zones de Sénart ou d'Évry, par exemple. Le port de Bonneuil-surMarne, beaucoup mieux situé pour desservir l'Est et le Sud de l'Île-de-France, n'est que très peu utilisé. La traversée de Paris limite le tirant d'air des convois à deux hauteurs de conteneurs, ce qui impose une réorganisation coûteuse des convois à Gennevilliers.

Le paradoxe économique et environnemental est complet puisque le transport fluvial s'effectue là où n'existe pas de problème majeur de congestion, entre Le Havre et les portes de Paris, alors que le transport routier est nécessaire et incontournable pour effectuer les derniers kilomètres dans l'aire urbaine dense. Cela démontre aussi par l'absurde à quel point les gains engendrés par la massification

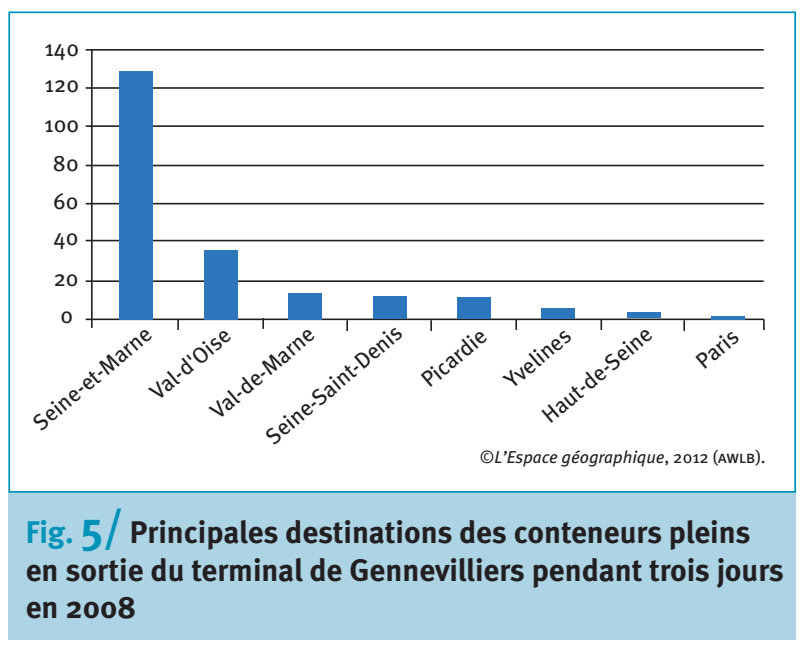




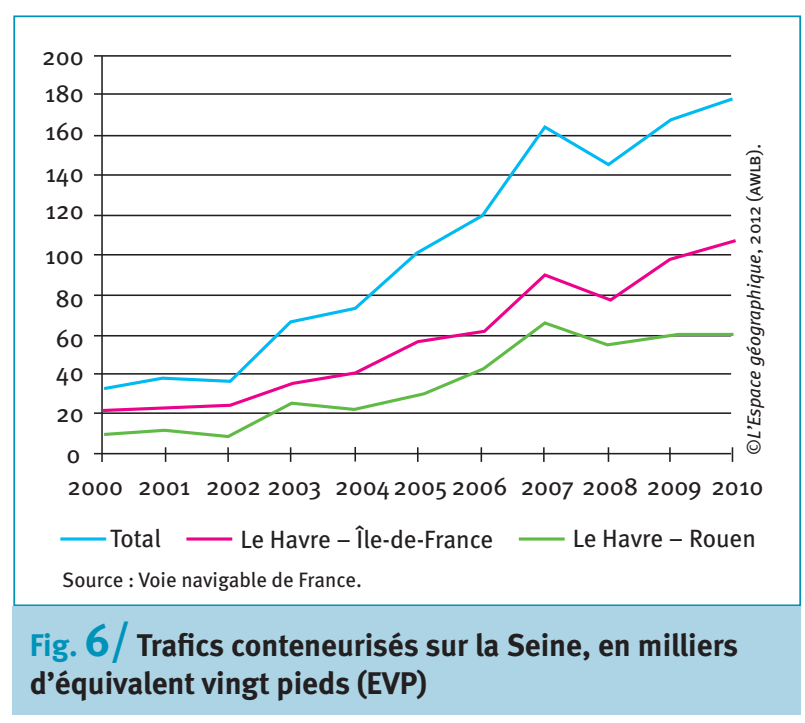

du transport fluvial sont élevés pour supporter de tels pré- et post-acheminements routiers tout en restant pertinents par rapport à une solution directe par la route entre Le Havre et la région parisienne (fig. 6). Par conséquent, le transport combiné fleuve-route, dans sa configuration actuelle, est lui aussi très fortement dépendant de la déconnexion spatiale entre le fleuve et la métropole. Dans ces conditions, sa part, malgré les succès enregistrés, ne peut rester que marginale.

\section{Reconnecter le fleuve à la logistique métropolitaine}

La reconnexion entre le fleuve et la logistique métropolitaine peut être envisagée à plusieurs échelles (fig. 7). La première est la petite échelle, celle des corridors qui mettent en relation la métropole avec les grandes portes d'entrée marimetropole avec les grandes portes d'entree mari-
à la logique diffuse du transport routier. L'Île-detime. Ces corridors s'opposent à la logique diffuse du transport routier. L'Île-deFrance dispose avec la Seine d'une ouverture maritime proche grâce aux ports de Rouen et du Havre, complémentaires dans la nature de leurs trafics. Cet axe fluvial, sans contrainte de gabarit ni de capacité jusqu'aux ponts de Paris, est un outil de massification exceptionnel, comme le montre l'exemple des conteneurs, en dépit de la courte distance (ci-dessus). Le canal Seine-Nord-Europe va permettre de désenclaver le bassin de la Seine. Il ouvre certes la possibilité d'une autoroute fluviale entre Anvers et la région parisienne. Inversement, il donne aussi aux acteurs rhénans, transitaires ou bateliers, l'occasion de s'intéresser au marché français, de s'y implanter, d'y apporter leur savoir faire, de dynamiser le transport fluvial sur la Seine actuellement entre les mains d'un nombre restreint d'entreprises en situation de rente, et cela en faveur des ports du Havre et de Rouen tant la proximité avec la région parisienne est évidente. Comme toute nouvelle infrastructure de transport, ce canal ouvre des marchés et des occasions à ceux capables de les saisir.

Plus complexe est la reconnexion à plus grande échelle, à celle de l'aire urbaine, pour aller vers un quatrième moment logistique (fig. 2, moment 4). Elle nécessite d'inventer un couple massification-dispersion des flux de marchandises reposant sur le fleuve et qui offre une alternative crédible par rapport à la route. Ce couple ne peut se limiter aux niches actuelles ni devenir véritablement métropolitain que s'il concerne aussi les produits manufacturés et alimentaires. Outre les conditions mises en évidence avec l'exemple des conteneurs, quelques pistes peuvent être dégagées en partant du constat suivant. Dans le cas d'un transport combiné fleuve-route, les deux seules variables d'ajustement permettant une baisse des coûts sont la taille des convois fluviaux plus les convois sont volumineux, plus le coût de la marchandise transportée est faible - et l'optimisation des pré- et post-acheminements à partir du terminal intérieur pour minimiser le coût du transport routier, la solution la plus radicale étant des entrepôts en bord à voie d'eau afin d'éviter tout transport routier.

L'identification et la valorisation de sites logistiques multimodaux (Allio, 2010) sont essentielles pour organiser l'offre logistique et la massification le long de l'axe Seine afin d'organiser le corridor à ses deux bouts. À la massification des trafics dans les ports 
maritimes doit répondre celle dans les métropoles. Un nombre très limité - une ou deux très grandes plates-formes de rang supérieur en Île-de-France pourrait jouer un rôle de $h u b$ fluvial et ferroviaire et proposer une offre logistique via la présence de grands entrepôts. Elles auraient une vocation nationale et européenne en étant raccordées aux itinéraires européens du fret, par le maillage des itinéraires ferroviaires et fluviaux. Elles pourraient assurer un rôle de redistribution des marchandises aux échelles internationales, régionales et locales, jusqu'à la distribution urbaine via des plates-formes secondaires. Le rééquilibrage de l'offre logistique passe par la création d'une de ces plates-formes de rang supérieur à l'ouest. Les projets élaborés dans le cadre du Grand Paris et du SDRIF envisagent à l'ouest de l'agglomération le site d'Achères (Blanc, 2010). Une seconde plate-forme de rang supérieur pourrait se situer à l'est-sud-est de l'agglomération

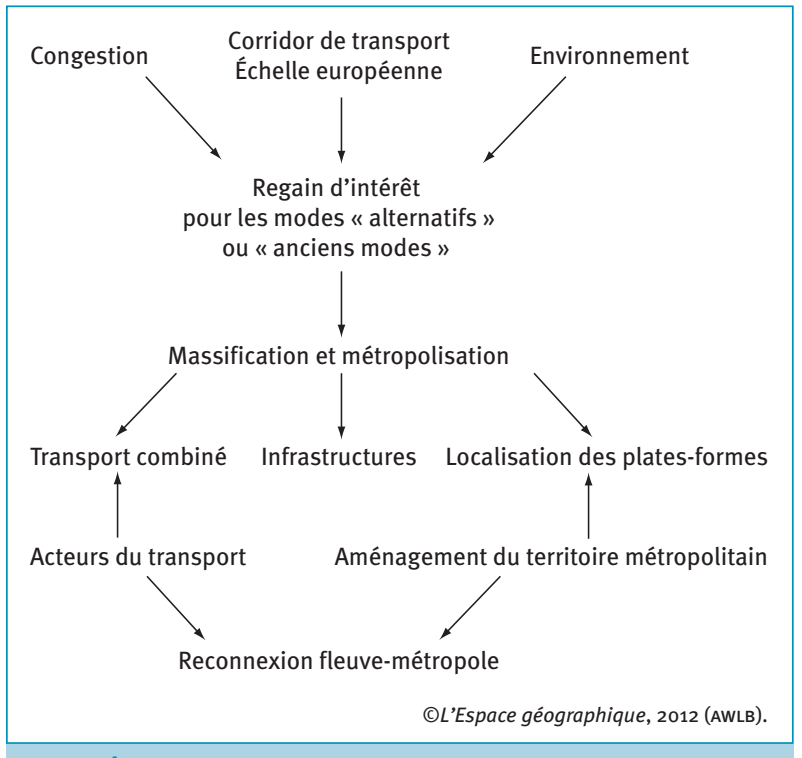

Fig. $7 /$ Vers une reconnexion du fleuve et de la ville? à proximité de la Francilienne, probablement vers Évry-Sénart.

De fortes réserves foncières sont nécessaires à leur mise en place, un peu à la façon d'un aéroport. Les sites existants sont sans doute trop exigus et trop insérés dans le tissu urbain pour jouer un tel rôle. Mais ils doivent être préservés avec une vocation plus régionale de gestion des flux de marchandises en étant bien reliés aux quelques plates-formes de rang supérieur. La localisation de ces dernières ne peut être sans doute que périphérique pour des questions de disponibilité foncière. Paradoxalement, pour répondre à l'étalement routier, un desserrement logistique de l'offre de transport combiné s'impose.

Reste à gérer les derniers kilomètres, ceux qui permettent d'amener les marchandises dans les rayons. Le fleuve peut encore jouer un rôle, comme il le fait pour les matériaux de construction, car il traverse l'ensemble de l'aire urbaine. Cette "logistique fluviale urbaine " reste largement à inventer en dépit de quelques expérimentations aux Pays-Bas, mais aussi à Paris. Elle nécessite des innovations pour charger/décharger des bateaux de palettes en zone urbaine dense, grâce à des quais à temps partagé, pouvant accueillir, en fonction des différents moments de la journée des promeneurs ou une activité de manutention, comme le fait aujourd'hui le Port de Paris sur le site de Tolbiac pour des trafics du BTP. Cela nécessite aussi de contenir la pression urbaine sur le fleuve à un moment où ce dernier redevient très attractif.

\section{Conclusion}

Une reconnexion entre le fleuve et la métropole est-elle possible pour le transport des marchandises, des grands flux internationaux qui arrivent par la voie maritime jusqu'aux derniers kilomètres, ceux de la logistique urbaine? Les limites des villes sont aujourd'hui tellement floues et l'imbrication et la hiérarchisation des flux sont telles 
qu'une esquisse de réponse ne peut être donnée qu'en replaçant le transport des marchandises dans les logiques métropolitaines auxquelles il s'intègre d'une façon systémique, en les subissant tout en y participant. Force est de constater la déconnexion radicale entre le fleuve et la ville en raison de l'efficacité du système routier à l'œuvre depuis des dizaines d'année. Ce système routier a abouti à une "périurbanisation logistique " dont les ressorts sont très similaires à ceux de l'habitat. Il participe à la mise en forme de la métropole: concentration à petite échelle, étalement et dispersion à l'échelle de l'aire urbaine, concentration à grande échelle sur des zones d'activités privilégiées situées d'abord à proximité immédiate des carrefours d'autoroutes et de rocades afin de garantir une très forte accessibilité, à nouveau à différentes échelles, de la desserte locale aux transports internationaux. Dans ce système routier métropolitain, le fleuve ne joue qu'un rôle marginal de niche qui n'a cessé de s'amenuiser depuis 40 ans. La métropole parisienne, par son poids qui amplifie les phénomènes et par la présence de la Seine, illustre cette déconnexion radicale et à l'inverse le rôle structurant du système routier sur son organisation et les formes de la métropole.

Le transport par la voie d'eau nécessite un certain nombre de conditions pour se développer: l'intérêt des acteurs du transport, des volumes, de la fiabilité, des prix inférieurs à ceux de la route. Une réelle fenêtre d'opportunité existe actuellement dans ce sens. Mais une véritable reconnexion du fleuve et de la métropole nécessite aussi de penser le couple massification-dispersion via l'aménagement de plates-formes logistiques multimodales, de rangs différents, de la très grande plate-forme accueillant des convois lourds et de très vastes entrepôts, localisée en périphérie, jusqu'au simple quai assurant des fonctions variées en temps partagé en zone urbaine dense,- l'ensemble fonctionnant en réseau et le transport routier assurant les derniers kilomètres. Cette reconnexion du fleuve et de la ville ne peut se concevoir que dans une stratégie volontariste d'aménagement des territoires de la logistique métropolitaine, à l'inverse de la "main invisible » du système routier.

\section{Références}

ALLIO R. (2010). Suivi des plates formes fluviales multimodales inscrites au SDRIF. État des lieux et perspectives de développement. Paris: Institut d'Aménagement et d'Urbanisme Île-de-France.

Beyer A., Debrie J. (2011). « Les temporalités frontalières et urbaines du port de Strasbourg. Analyse géohistorique d'une relation fluviale ville-port ». Métropole, $n^{0} 10$.

http://metropoles.revues.org/4494

Blanc C. (2010). Le Grand Paris du xxle siècle. Paris: Le Cherche Midi, 262 p.

BOWEN JR. J.T. (2008). " Moving places: the geography of warehousing in the US ». Journal of Transport Geography, vol. 16, nº 6, p. 379-387.

CIDELL J. (2010). "Concentration and decentralization: the new geography of freight distribution in US metropolitan areas ». Journal of Transport Geography, vol. 18, n 3, p. 363-371.

COMBES P.-P., MAYER T., THISSE J.-F. (2006). Économie géographique. L'intégration des régions et des nations. Paris : Economica, coll. Corpus «Économie», 397 p.

Dablanc L. (2007). «Goods transport in large European cities: Difficult to organize, difficult to modernize ». Transportation Research Part A: Policy and Practice, vol. 41, nº 3, p. $280-285$. 
Dablanc L., Rakotonarivo D. (2010). « The impacts of logistics sprawl: How does the location of parcel transport terminals affect the energy efficiency of goods' movements in Paris and what can we do about it? ». Procedia - Social and Behavioral Sciences, vol. 2, nº 3, p. 6087-6096.

Direction régIonale de l'Équipement d’Île-de-France (Dreif)(2007). « Le fret en Île-de-France. Statistiques 2006 ». Paris : DrelF, note de synthèse, $4 \mathrm{p}$.

ELISSALDE B. « Métropolisation». Hypergeo. http://www.hypergeo.eu/spip.php?article75

Frémont A., Franc P. (2010). « Hinterland transportation in Europe: Combined transport versus road transport ». Journal of Transport Geography, vol. 18, p. 548-556.

Frémont A., Franc P., Slack B. (2009). "Desserte fluviale des ports maritimes et transport de conteneurs: quels enjeux pour les ports français du Havre et de Marseille dans le contexte européen? ». Cybergéo: European Journal of Geography, article 437. http://www.cybergeo.eu/index21743.html.

Graille F., Prie Y. (2009). «Approfondissement de l'estimation régionale du parc d'entrepôts en île-de-France ». Paris : Direction régionale de l'équipement d'île-de-France, novembre, $16 \mathrm{p}$.

Grumbach A. (2009). Seine Métropole. Paris Rouen Le Havre. Le diagnostic prospectif de l'agglomération parisienne. Paris: Archibooks-Sautereau, $191 \mathrm{p}$.

Gullbault M. (2008). Enquête Echo. "Envois-Chargeurs-Opérations de transport». Résultats de référence. Paris: Lavoisier, coll. «INRETS », n 56, 205 p.

Hall P.V., Clark A. (2010). « Maritime ports and the politics of reconnection ». In DeSFOR G, Laidley J., Stevens Q., Schubert D. (eds), Transforming Urban Waterfronts. Fixity and Flow. Abingdon: Routledge, p. 17-34.

Hesse M. (2008). The City as a Terminal. The Urban Context of Logistics and Freight Transport. Ashgate: Aldershot, Series Transport and Mobility, 224p.

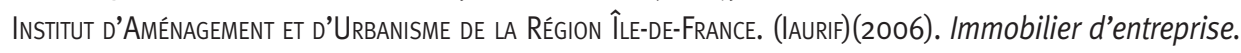
Nouvelle géographie, nouvelles stratégies. Paris : La Documentation française, coll. «Les Cahiers de l'IAURIF» n' $145,176 \mathrm{p}$.

Institut d’Aménagement et d’Urbanisme de la Région Île-de-France. (Iaurif)(2008). La Place de l'île-deFrance dans l'hinterland portuaire du Havre : le maillon fluvial. Paris : IAURIF, rapport, $66 \mathrm{p}$.

Lalandre A. (2004). Histoire des ports de Paris et de l'île-de-France. Rennes: Éditions OuestFrance, coll. «Histoire», $127 \mathrm{p}$.

0'Connor K. (2010). "Global city regions and the location of logistics activity ». Journal of Transport Geography, vol. 18, nº 3, p. 354-362.

PAFFonI E. (2011), « Localisation urbaine et spécialisation fluviale des sites portuaires en île-deFrance ", soumis prochainement à la revue Mappemonde.

SAVY M. (2006a). Logistique et territoire. Paris: La Documentation française, 63 p.

SAvY M. (2006b). Le Transport de marchandises. Paris: Eyrolles, 372 p. 